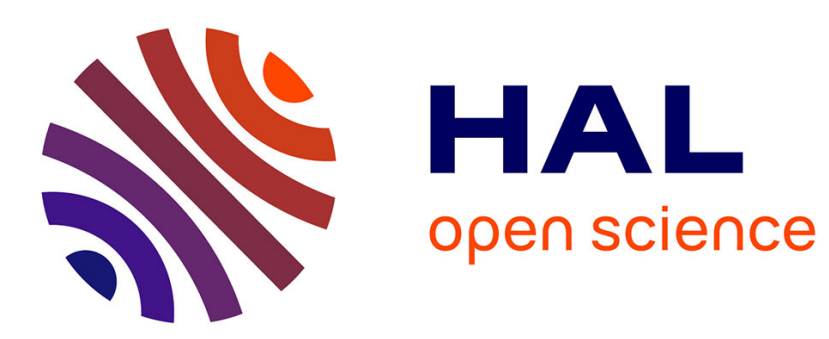

\title{
Ecoulement à surface libre dans un massif de sol soumis à la marée: comparaison théorie-expérimentation
}

\author{
A. Rezzoug, Alain Alexis, Pierre Thomas
}

\section{To cite this version:}

A. Rezzoug, Alain Alexis, Pierre Thomas. Ecoulement à surface libre dans un massif de sol soumis à la marée: comparaison théorie-expérimentation. Canadian Geotechnical Journal, 1999, 36 (1), pp.13-20. 10.1139/t98-078 . hal-01006762

\section{HAL Id: hal-01006762 \\ https://hal.science/hal-01006762}

Submitted on 3 Feb 2018

HAL is a multi-disciplinary open access archive for the deposit and dissemination of scientific research documents, whether they are published or not. The documents may come from teaching and research institutions in France or abroad, or from public or private research centers.
L'archive ouverte pluridisciplinaire HAL, est destinée au dépôt et à la diffusion de documents scientifiques de niveau recherche, publiés ou non, émanant des établissements d'enseignement et de recherche français ou étrangers, des laboratoires publics ou privés. 


\title{
Écoulement à surface libre dans un massif de sol soumis à la marée : comparaison théorie-expérimentation
}

\author{
A. Rezzoug, A. Alexis et P. Thomas \\ Laboratoire de Génie Civil de Nantes - Saint-Nazaire, École Centrale de Nantes - Université de Nantes, Institut \\ Universitaire de Technologie, B.P. 420, 44606 Saint-Nazaire, France.
}

\begin{abstract}
Résumé : Les interactions eau-sol conditionnent le comportement des ouvrages tout particulièrement dans les zones littorales ou estuariennes comportant de nombreux aménagements portuaires ou touristiques. L'équilibre des rives et des ouvrages est perturbé par les variations cycliques du régime hydraulique engendrées par la marée. L'objet de cet article est l'étude de l'écoulement à surface libre dans un massif soumis à la marée. Une démarche scientifique globale est proposée qui associe étroitement modélisation et expérimentations en laboratoire et in situ. La confrontation de ces différentes approches montre un bon accord global des résultats, l'existence d'une zone perturbée subissant des variations importantes de vitesse et de pression, et l'existence d'un niveau d'équilibre à l'intérieur du massif, supérieur au niveau moyen de la marée et proche de la pleine mer. Cette démarche menant de front modélisation et expérimentation permet d'obtenir un ensemble de résultats originaux pouvant présenter un grand intérêt pour la connaissance du comportement des zones intertidales.
\end{abstract}

Mots clés : sol, marée, massif, écoulement, modèle, essais en laboratoire, essais in situ.

\begin{abstract}
Water-soil interactions affect the behaviour of structures, especially in coastal or estuarine areas with many harbours and tourism amenities. The equilibrium between banks and structures is disturbed by periodical variations of tidal flow. The purpose of this paper is the study of free surface flow in a tidal bank. A global scientific approach is proposed that closely associates modeling, laboratory, and in situ tests. The comparison of these approaches demonstrates a good overall agreement of results, the existence of a disturbed area that undergoes important variations in velocities and pressures, the existence of an equilibrium level within the embankment higher than the mean tide level and close to the high tide level. This modelling and experimental approach allows us to obtain a set of original results, of tremendous potential interest, leading to the understanding of the behaviour of intertidal areas.
\end{abstract}

Key words: soil, tide, embankment, flow, model, laboratory tests, in situ tests.

\section{Introduction}

L'étude des interactions eau-sol constitue un domaine essentiel de la géotechnique. Ces interactions sont aussi diverses qu'importantes, tout particulièrement dans les milieux frontières littoraux ou estuariens, marqués par l'importance des ouvrages d'aménagement portuaire ou touristique. Ainsi, l'étude du comportement des massifs soumis à la marée revêt une grande importance pour la prévision des risques d'instabilité et pour l'analyse de la localisation et du dimensionnement des ouvrages.

Le comportement de ces sols et ouvrages est fonction du régime hydraulique cyclique créé par la marée et caractérisé par une alternance de remplissage et de vidange du massif. Ces phénomènes engendrent des perturbations (rupture de pente, affouillement) affectant directement l'équilibre des rives et des ouvrages d'aménagement (Chapon 1966; De Cazenove 1971). La modélisation de l'écoulement dû à la marée a été abordée analytiquement à l'aide d'une formulation linéarisée simple par Marino (1973), Rat et Vautrin (1973) et Razack et al. (1980). Toutefois la méthode de résolution a été critiquée par Vauclin (1975). En outre l'observation courante des ingénieurs portuaires semble indiquer l'établissement d'un niveau d'eau dans les quais et talus estuariens nettement supérieur au niveau moyen de la marée (plus de $1 \mathrm{~m}$ ). Cette surcote est d'ailleurs prise en compte dans les calculs d'ouvrages.

Cependant la recherche bibliographique effectuée n'a pas révélé de publications plus récentes sur l'écoulement dû à la marée dans un talus.

L'objectif de cet article est l'étude de l'écoulement à surface libre dans un massif de sol soumis à la marée. La méthodologie adoptée repose sur la complémentarité des trois approches suivantes : (i) modélisation théorique, numérique, (ii) expérimentation en laboratoire et sur un massif naturel, (iii) confrontation théorie-expérimentations. 
Fig. 1. Schéma du talus soumis au marnage.

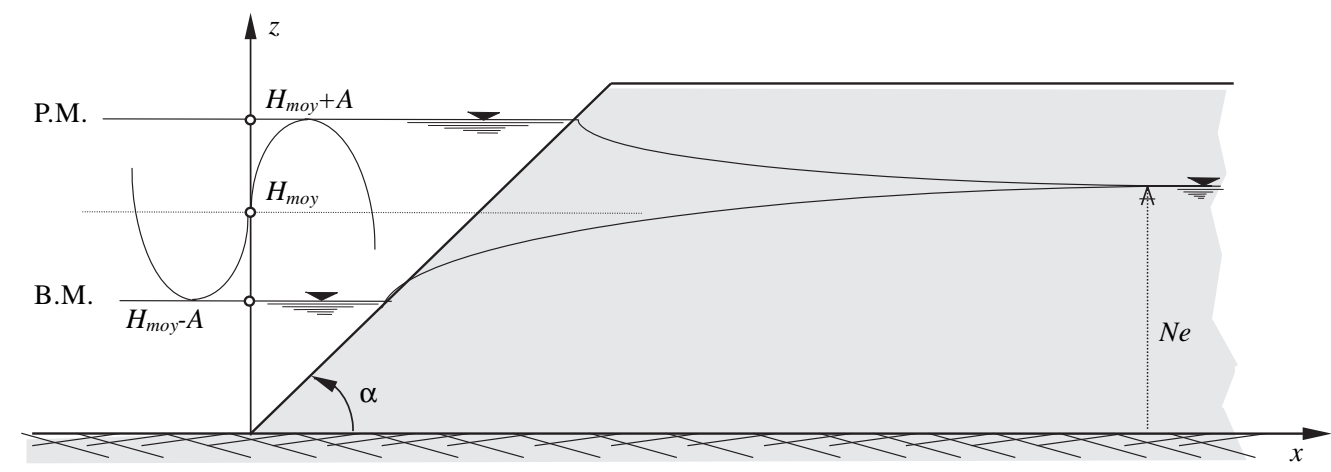

\section{Modélisation théorique et résolution numérique}

Modélisation de l'écoulement transitoire dans les sols

Un massif de sol, soumis à un écoulement transitoire, est caractérisé par la coexistence de zones d'écoulement en pression et en dépression. La frontière entre ces deux zones, soumise à la pression atmosphérique, constitue la surface libre. Au-dessus de cette surface, la pression interstitielle varie linéairement dans la zone non saturée constituant la frange capillaire. La zone située en-dessous de la surface libre est saturée et en pression. (Humbert 1984; Zaradny 1993).

La théorie des milieux saturés, où la surface libre est considérée comme limite supérieure de l'écoulement, repose sur la description de l'écoulement dans l'ensemble du domaine par l'équation :

$$
\operatorname{div}(\overline{\bar{k}} \stackrel{\rightarrow}{\operatorname{grad} h})=S \frac{\partial h}{\partial t}
$$

où $\overline{\bar{k}}$ est le tenseur de perméabilité au sens de Darcy, $h$ la charge hydraulique et $S$ le coefficient d'emmagasinement spécifique.

En posant l'hypothèse de Dupuit de quasi-verticalité des équipotentielles, Polubarinova (1962) a étudié la vidange rapide des barrages et a proposé une équation des écoulements non permanents dans les milieux isotropes indéformables qui s'écrit en conditions planes dans le plan vertical $(\mathrm{x}, \mathrm{z})$ :

$$
\frac{\partial^{2} H^{2}(x, t)}{\partial x^{2}}=\frac{2 n}{k} \frac{\partial H(x, t)}{\partial t}
$$

où $H(x, t)$ est la hauteur de la surface libre, $n$ la porosité efficace du sol et $t$ le temps.

Cette formulation du mouvement de la surface libre est l'équation de Dupuit en régime transitoire non linéaire (Vauclin 1975; Ould Amy 1990; Zaradny 1993). La validité de la loi de Darcy dans le domaine transitoire cyclique lent, qui est celui de la marée, ne semble pas remise en cause (Hall et al. 1994).

\section{Modélisation d'un écoulement cyclique à surface libre dans un talus soumis à la marée}

L'onde excitatrice de la marée est complexe, son caractère pseudo-périodique résultant de la superposition d'oscillations de périodes différentes (semi-diurne ou diurne, lu- naire, annuelle). Pour réduire la complexité du problème, nous effectuons les hypothèses de : (i) périodicité de la marée en ne gardant que sa composante principale semidiurne sinusoïdale(de période $T$ voisine de $12 \mathrm{~h}$ ), d'amplitude $A$, de niveau moyen $H_{\text {moy }}$ (mesurée à partir du substratum), et de pulsation $\omega$ supposés constants, (ii) homogénéité et incompressibilité du sol constituant le massif, (iii) imperméabilité du substratum sur lequel il repose, (iv) réductibilité du problème à un cas plan; les génératrices du talus sont orthogonales à la direction de propagation.

La forme sinusoïdale de l'onde marée incidente (fig.1) permet d'écrire la condition à la limite :

$$
\text { [3] } \quad H(x=0, t)=H_{\text {moy }}+\mathrm{A} \sin \omega t
$$

À l'intérieur du talus, l'oscillation amortie du niveau $H(x, t)$ de la surface libre est solution de [2] indiquée au paragraphe précédent. Le niveau de nappe à $x$ infini n'est pas imposé.

Le cas du massif vertical $(\alpha=\pi / 2)$ représente un cas pratique moins fréquent de massif retenu par un écran, de perméabilité très supérieure à celle du massif (palplanches perméables). Ce cas sera utilisé uniquement par la suite comme cas limite. L'expression mathématique de [2] et la forme périodique de [3] permettent de montrer que la moyenne quadratique du niveau de la surface libre est constante (dans le temps et dans l'espace). Dans le cas du massif vertical, l'égalisation de cette moyenne quadratique obtenue à $x=0$ et à $x$ infini aboutit au résultat analytique important (Alexis 1987) :

$$
N_{\mathrm{e}}=\sqrt{H_{\text {moy }}^{2}+\frac{A^{2}}{2}}
$$

Ce résultat montre que le niveau d'équilibre $N_{\mathrm{e}}$ atteint dans le massif peut être supérieur au niveau moyen de la marée. Physiquement, ce résultat quelque peu surprenant peut s'expliquer par le fait que la surface offerte à l'écoulement entrant dans le massif est supérieure à celle offerte à l'écoulement sortant. L'écoulement cyclique est ainsi susceptible de produire une accumulation d'eau dans le massif.

\section{Méthodes de simulation numérique}

Méthode basée sur la résolution de l'équation non linéaire en différences finies

Nous avons effectué la résolution numérique de l'équation de Dupuit non linéaire en régime transitoire [2] avec la 
Fig. 2. Procédure de la résolution semi-analytique.

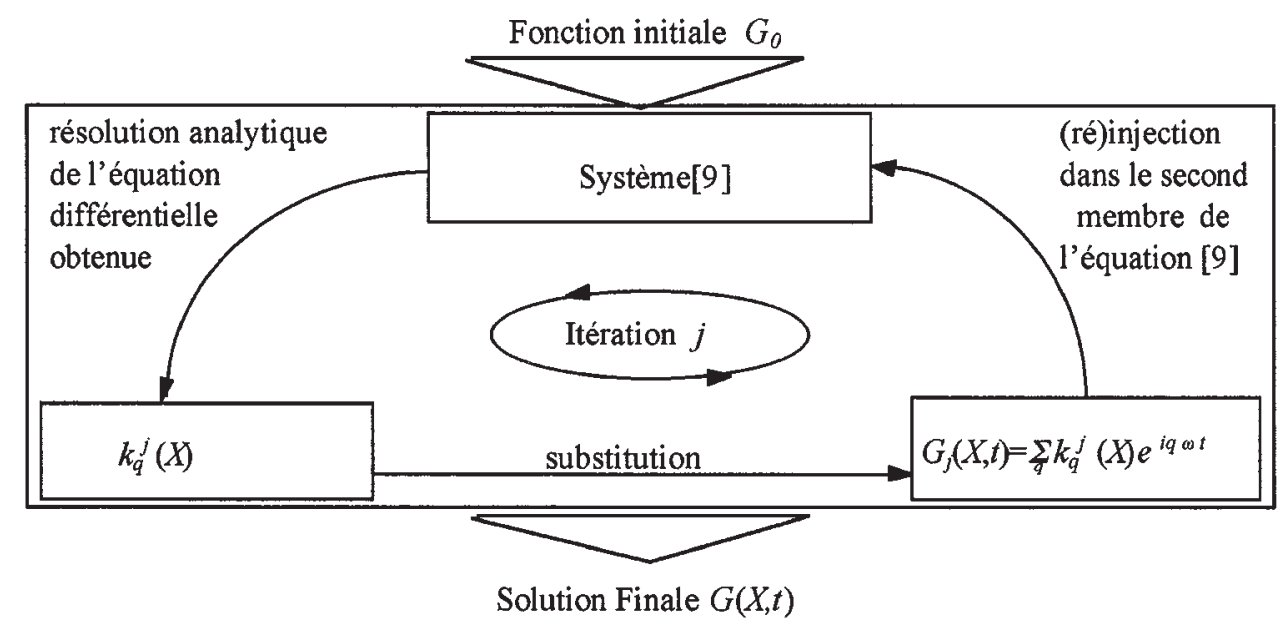

condition à la limite [3]. Pour simplifier la discrétisation de la pente du talus, nous avons transformé ces équations au moyen d'un changement de variable et de la fonction de translation horizontale $a(t)$ :

$$
X=x-a(t) \quad \text { avec } \quad a(t)=\frac{1}{\tan \alpha}\left(H_{\mathrm{moy}}+A \sin \omega t\right)
$$

Nous obtenons ainsi une nouvelle forme de l'équation de Dupuit avec des conditions aux limites plus simples :

$$
\left\{\begin{array}{l}
\frac{\partial H(X, t)}{\partial t}=\frac{k}{2 n} \frac{\partial^{2} H^{2}(X, t)}{\partial X^{2}}-\frac{A \omega}{\tan \alpha} \cos \omega t \frac{\partial H(X, t)}{\partial X} \\
H(X, t=0)=H_{\text {moy }} \\
H(X=0, t)=H_{\text {moy }}+A \sin \omega t
\end{array}\right.
$$

À partir des conditions initiales, et de l'expression en différences finies de l'équation différentielle de [6], on a décrit plusieurs centaines de cycles jusqu'à l'établissement de la périodicité du phénomène.

Le choix du pas de temps a été effectué par analyse de convergence et de stabilité (Rezzoug 1994), ce qui conduit, dans le cas limite du massif vertical, à :

$$
\frac{k}{2 n} \frac{\Delta t}{(\Delta x)^{2}}=\frac{1}{4\left(H_{\text {moy }}+A\right)}
$$

où $\Delta t$ et $\Delta x$ sont respectivement les incréments de temps et d'abscisse.

\section{Méthode semi-analytique}

Afin de vérifier les résultats obtenus par la méthode précédente, on a proposé une méthode basée sur la résolution analytique, qui repose sur la linéarisation de l'équation non linéaire de Dupuit en régime transitoire [2] et sur le calcul des coefficients de Fourier du développement de la solution périodique.

La linéarisation simple due à Boussinesq (1904) (et exploitée depuis par plusieurs auteurs : Castany 1967; Dagan 1967; Desai 1970, 1971; Rat et Vautrin 1973; Marino 1973; Razack et al. 1980; Zaradny 1993) ne permet pas de retrou- ver la surélévation du niveau d'équilibre indiquée par le résultat analytique [4].

Nous utilisons une forme mieux adaptée, suggérée par Polubarinova (1962), de linéarisation quadratique de [2] :

$$
\left\{\begin{array}{l}
X=x-a(t) \\
G(X, t)=H^{2}(x, t)
\end{array}\right.
$$

Le système à résoudre prend alors la forme suivante :

$$
\left\{\begin{array}{c}
\frac{\partial G(X, t)}{\partial t}-c \frac{\partial^{2} G(X, t)}{\partial X^{2}}=d \cos \omega t \frac{\partial G(X, t)}{\partial X} \\
\text { conditions : }\left\{\begin{array}{c}
G(X=0, t)=\left(H_{\text {moy }}+A \sin \omega t\right)^{2} \\
G(X \rightarrow+\infty, t)=\text { constante } \\
G(X, t+T)=G(X, t)
\end{array}\right.
\end{array}\right.
$$

avec : $c=\frac{k H_{\text {moy }}}{n}$, et $d=\frac{A \omega}{\tan \alpha}$.

Le mouvement étant oscillatoire amorti, la solution est développable en série de Fourier. Les valeurs des coefficients sont obtenues par résolution itérative d'équations différentielles selon la procédure schématisée à la figure 2 .

Le processus est initialisé par l'introduction d'une fonction connue telle que $G_{\mathrm{o}},(j=0)$ dans [9], qui permet l'obtention des coefficients de Fourier, correspondant à la première itération $(j=1)$. La solution ainsi obtenue $G_{1}(X, t)$ est injectée dans le second membre de [9], et le processus est itéré jusqu'à obtention de la solution finale $G_{1}(X, t)$.

Cette méthode semi-analytique conduit à l'expression complète du carré de la solution :

$$
\begin{aligned}
& H^{2}(x, t)= \\
& \sum_{q}\left\|K_{p, q}\right\| e^{-\Re \lambda_{p}(x-a(t))} \cos \left\{q \omega t-\mathfrak{I} \lambda_{p}[x-a(t)]+\phi_{p, q}\right\}
\end{aligned}
$$

$\left\|K_{p, q}\right\|$ et $\phi_{p, q}$ sont respectivement le module et l'argument du complexe $K_{p, q}$, dont les valeurs peuvent être obtenues par un calcul itératif sur un tableau de dimension $p \times q$, (Rezzoug et al. 1994b); les entiers relatifs $p$ et $q$ varient dans l'intervalle $(\ldots,-1,0,+1, \ldots) ; \lambda_{p}$ est la racine carrée du 
Fig. 3. Surfaces libres d'écoulement dans un massif vertical lors du flot.

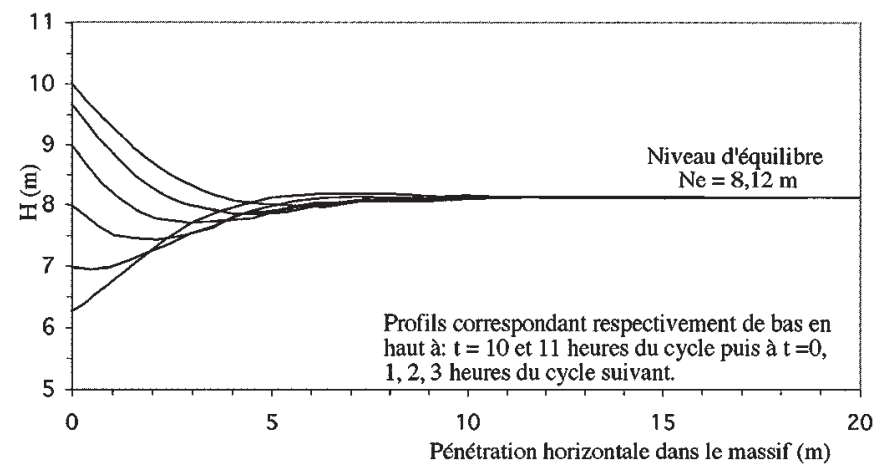

complexe $i(p \omega / c)$, elle s'écrit aussi en fonction de sa partie réelle et sa partie imaginaire $\lambda_{p}=\mathfrak{R} \lambda_{p}+i \mathfrak{I} \lambda_{p} ; i$ est le nombre imaginaire pur. La fonction [10] décrit la ligne de surface libre à tout instant du cycle marée. Elle montre une onde oscillatoire qui se propage (terme sinusoïdal) et s'amortit progressivement (terme exponentiel) autour d'un niveau constant.

Dans le cas limite du massif vertical, [10] se réduit à :

$$
\begin{aligned}
& H^{2}(x, t)= \\
& H_{\text {moy }}^{2}+\frac{A^{2}}{2}+2 A H_{\text {moy }} \mathrm{e}^{-\Re \lambda_{1} x} \sin \left(\omega t-\mathfrak{I} \lambda_{1} x\right) \\
& \quad-\frac{A^{2}}{2} \mathrm{e}^{-\Re \lambda_{2} x} \cos \left(2 \omega t \mathfrak{I} \lambda_{2} x\right)
\end{aligned}
$$

$\operatorname{avec} \mathfrak{R} \lambda_{1}=\mathfrak{I} \lambda_{1}=\sqrt{\frac{\omega}{2 c}}=\sqrt{\frac{\pi n}{T k H_{\text {moy }}}}$

et $\mathfrak{R} \lambda_{2}=\mathfrak{I} \lambda_{2}=\sqrt{\frac{\omega}{c}}=\sqrt{\frac{2 \pi n}{T k H_{\text {moy }}}}$

Le niveau d'équilibre peut être alors calculé par passage à la limite :

$$
\begin{aligned}
& \lim H^{2}(x \rightarrow+\infty, t)=H_{\text {moy }}^{2}+\frac{A^{2}}{2} \\
& \Rightarrow \quad N_{\mathrm{e}}=\sqrt{H_{\text {moy }}^{2}+\frac{A^{2}}{2}}
\end{aligned}
$$

Nous retrouvons ainsi le résultat analytique important [4] : une surélévation du niveau d'équilibre $N_{\mathrm{e}}$ (constant) par rapport au niveau moyen $H_{\text {moy }}$ de la marée.

L'existence de cette surélévation permet de confirmer semi-analytiquement les résultats obtenus par le traitement de [2] non linéarisée en différences finies.

Les deux méthodes donnant les mêmes résultats, à $0,1 \%$ près (Rezzoug 1994), le risque d'erreurs cumulées par un nombre important de cycles de calcul en différences finies est donc négligeable. Cette dernière méthode a été conservée par la suite, car elle permet de prendre en compte des conditions aux limites plus adaptées aux diverses applications.
Fig. 4. Surfaces libres d'écoulement dans un talus chaque heure du cycle de marée (origine à l'intersection talus-substratum).

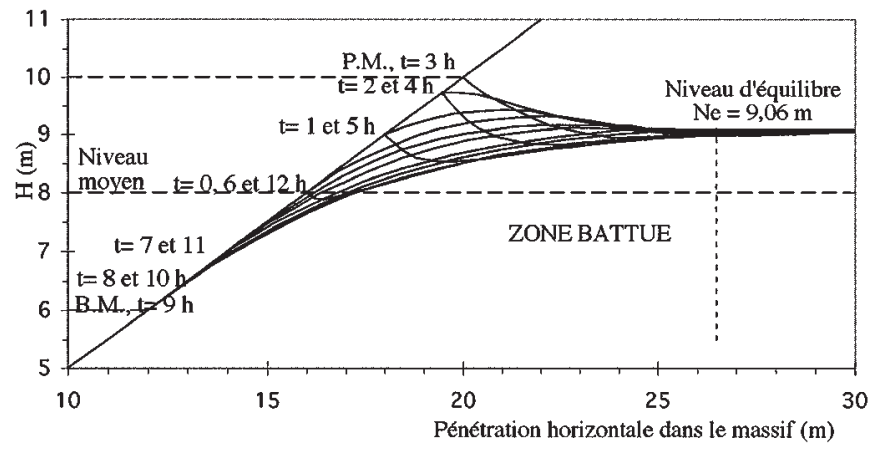

La résolution de l'équation de Dupuit appliquée à un massif de sol soumis à un cycle de marée conduit à un ensemble de résultats synthétisé graphiquement sur les figures 3 à 6 .

La marée semi-diurne $(T=12 \mathrm{~h})$ est composée de la phase de marée montante (flot) de basse mer (B.M.) à pleine mer (P.M.) et de la phase de marée descendante (jusant) de P.M. à B.M. L'instant de mi-flot est choisi comme temps origine. Les instants caractéristiques sont donc : mi-flot $(t=0)$, P.M. $(t=3 \mathrm{~h})$, mi-jusant $(t=6 \mathrm{~h})$, B.M. $(t=9 \mathrm{~h})$.

La figure 3 montre la montée de la surface libre lors du flot dans un massif vertical (sol : limon de perméabilité $2,2 \times 10^{-5} \mathrm{~m} / \mathrm{s}$ et de porosité 0,4 ; marée de caractéristiques $\left.T=12 \mathrm{~h} ; H_{\text {moy }}=8 \mathrm{~m} ; A=2 \mathrm{~m}\right)$. Les courbes sont représentées à toutes les heures du flot de la basse mer (B.M.) à la pleine mer (P.M.). Elles tendent toutes vers un niveau d'équilibre constant qui dépasse ici le niveau moyen de $12 \mathrm{~cm}$ environ (ce qui est en accord avec le résultat [4]). Nous constatons qu'au-delà des premiers $3 \mathrm{~m}$ du côté terre la ligne de surface libre est proche de l'horizontale, ce qui valide l'hypothèse de Dupuit.

La figure 4 synthétise les profils de surface libre à toutes les heures d'un cycle de marée dans un talus homogène de pente $1 / 2$, $\left(\alpha\right.$ voisin de $\left.26^{\circ}\right)$. L'ensemble des courbes représente la zone balayée par la surface libre durant une période de marée. Ce diagramme met en évidence le domaine de «zone battue », situé entre la ligne supérieure du diagramme et le substratum. Des phénomènes cycliques à caractère dynamique s'y produisent et peuvent causer des altérations du sol. La longueur de la zone battue est définie par la dimension horizontale du domaine (dans le talus) à l'intérieur duquel l'amplitude de fluctuation de la surface libre est supérieure à $10 \%$ de l'amplitude de la marée. La comparaison avec la figure 3 permet de mettre en évidence l'augmentation de la surélévation du niveau d'équilibre avec la décroissance de la pente du talus. Cette surélévation est ici de $1,06 \mathrm{~m}$, alors qu'elle était de $0,12 \mathrm{~m}$ dans le cas vertical. En effet l'écart entre la surface offerte à l'écoulement entrant et la surface offerte à l'écoulement sortant augmente quand la pente s'adoucit.

La figure 5 représente les diagrammes enveloppes dans un massif vertical pour quatre types de sols et toujours pour la même marée. Sur ces courbes, la longueur de la zone battue pour chacun des quatre types de sols est de $2,5 \mathrm{~m}$ pour l'argile, 6,0 m pour le limon sableux, 9,5 m pour le sable fin, $18,0 \mathrm{~m}$ pour le sable moyen. 
Fig. 5. Diagramme enveloppe pour quatre types des sols lors d'un cycle complet.

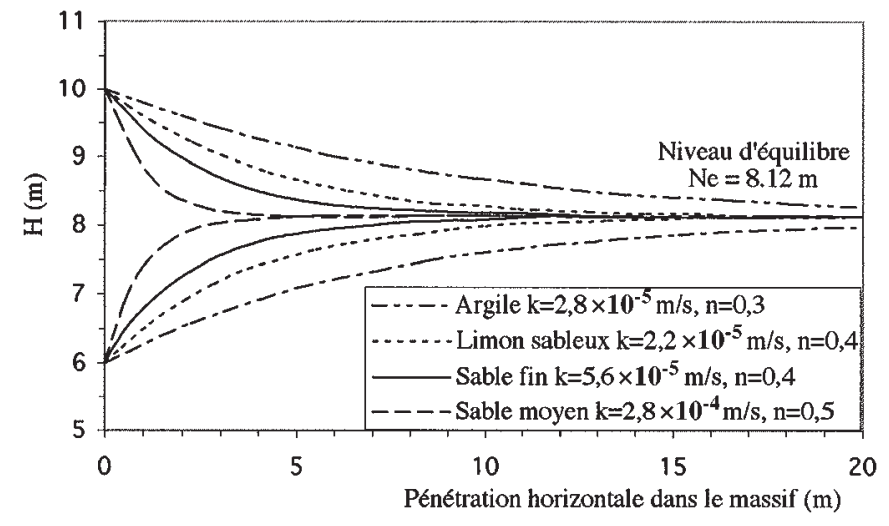

Relativement faible dans les sols fins peu perméables, la longueur de la zone battue devient importante dans les sols de perméabilité conséquente. Cette distance est estimée, dans le cas limite du massif vertical, à une valeur proche de $800(k / n)^{1 / 2}($ en m).

La figure 6 montre la surélévation du niveau d'équilibre $\left(\Delta N_{\mathrm{e}}=N_{\mathrm{e}}-H_{\mathrm{moy}}\right)$, en fonction de l'inclinaison $\alpha$ du talus, pour la même marée (amplitude $A=2 \mathrm{~m}$, niveau moyen $H_{\text {moy }}=8 \mathrm{~m}$ ) et pour divers types de sol. Ces sols sont caractérisés par des fourchettes de valeurs du rapport $k / n$ (en $\mathrm{m} / \mathrm{s})$ : sables $3,3 \times 10^{-2}$ à $1,1 \times 10^{-4}$; limons sableux $1,4 \times$ $10^{-4}$ à $6,6 \times 10^{-6}$; limons $2,9 \times 10^{-5}$ à $1,0 \times 10^{-6}$; argiles $1,2 \times 10^{-5}$ à $9,1 \times 10^{-8}$. Le point $\left(90^{\circ} ; 0,12 \mathrm{~m}\right)$ montre que pour un massif vertical, la surélévation de l'eau au-dessus du niveau moyen de la marée n'est que $\Delta N_{\mathrm{e}}=12 \mathrm{~cm}$, et indépendante du type de sol, alors qu'elle est proche de $2 \mathrm{~m}$ (valeur de l'amplitude) dans le cas des pentes faibles. Elle est plus importante dans le cas des argiles que dans le cas des sables.

Dans ce problème de modélisation des écoulements dans un massif soumis à des sollicitations cycliques, les modélisations théoriques et les résolutions reposent sur des hypothèses, des conditions aux limites et des calculs dont il convient de vérifier la validité au moyen d'expérimentations en laboratoire et in situ, prenant en compte la spécificité et la variabilité des phénomènes réels.

\section{Mise en place d'outils expérimentaux de validation}

\section{Modélisation physique}

La spécificité des écoulements à surface libre dans des massifs sous sollicitations hydrauliques cycliques, et l'absence de description de dispositifs expérimentaux dans la bibliographie, conduisent à construire un modèle physique bidimensionnel simple de conception originale.

La réalisation de cette modélisation physique a visé plusieurs objectifs : (i) simuler la propagation de la marée dans des massifs, (ii) effectuer un suivi de l'évolution de la surface libre et des pressions interstitielles dans le massif, (iii) mesurer la perméabilité du massif en place, (iv) permettre l'expérimentation avec différents types de marées et de sols.
Fig. 6. Surélévation du niveau d'équilibre en fonction de l'inclinaison du talus pour différents types de sols.

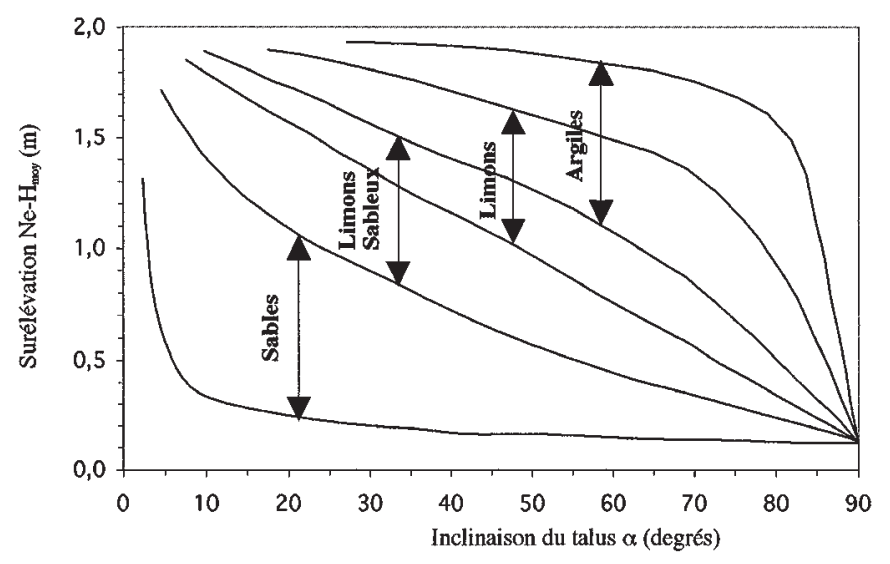

Les résultats complets de l'expérimentation sur le modèle physique ont été publiés antérieurement (Rezzoug et al. $1994 a)$.

Le système expérimental (fig. 7) est composé de deux parties principales : l'excitateur (simulation de la marée) et le récepteur (domaine de l'expérimentation).

Le domaine d'expérimentation est constitué, dans son ensemble, d'un bac de mesures de forme parallélépipèdique de dimensions $1,3 \times 1,18 \times 0,2 \mathrm{~m}$. Ce bac repose sur une base horizontale imperméable et contient une tranche de sol comprise entre deux parois latérales en plexiglas. Ces deux parois limitent également deux réservoirs latéraux ayant la profondeur du bac, et communiquant directement avec le sol à travers des grilles. Sur le réservoir (1) aval la simulation du phénomène de la marée est appliquée, alors que sur le réservoir (2) amont le niveau d'eau est laissé libre. Ce dernier est exploité pour l'observation et la simulation de la limite infinie du massif.

Les mesures de charges sont effectuées à l'aide de tubes piézométriques connectés aux prises de pressions. Ces prises sont installées de manière à former un maillage rectangulaire régulier sur toute une face du modèle. Les conditions aux limites naturelles ont été simulées en établissant une nappe à surface libre et en faisant varier le niveau pour représenter l'impact de la marée. L'évolution de la surface libre et le champ de pression dans la zone saturée ont été suivis lors des oscillations périodiques de la nappe.

Deux campagnes de mesure ont été effectuées, une sur une tranche de sol à écran vertical et l'autre simulant un talus de pente unité. La réalisation des mesures a conduit dans les deux cas aux observations suivantes :

(i) La périodicité : l'oscillation de l'onde incidente sinusoïdale (côté mer) est transmise à la surface libre à l'intérieur du massif, qui oscille avec la même période $T$;

(ii) La zone de perturbation : les fluctuations de la nappe engendrent des changements importants et alternatifs du champ de pression dans la zone battue;

(iii) Le niveau d'équilibre : le niveau d'eau dans le réservoir côté terre est stable et supérieur au niveau moyen côté mer.

La complexité et la diversité des phénomènes naturels intervenant dans les écoulements à surface libre dans les massifs littoraux ou estuariens soumis à des sollicitations 
Fig. 7. Schéma général du modèle physique expérimental.

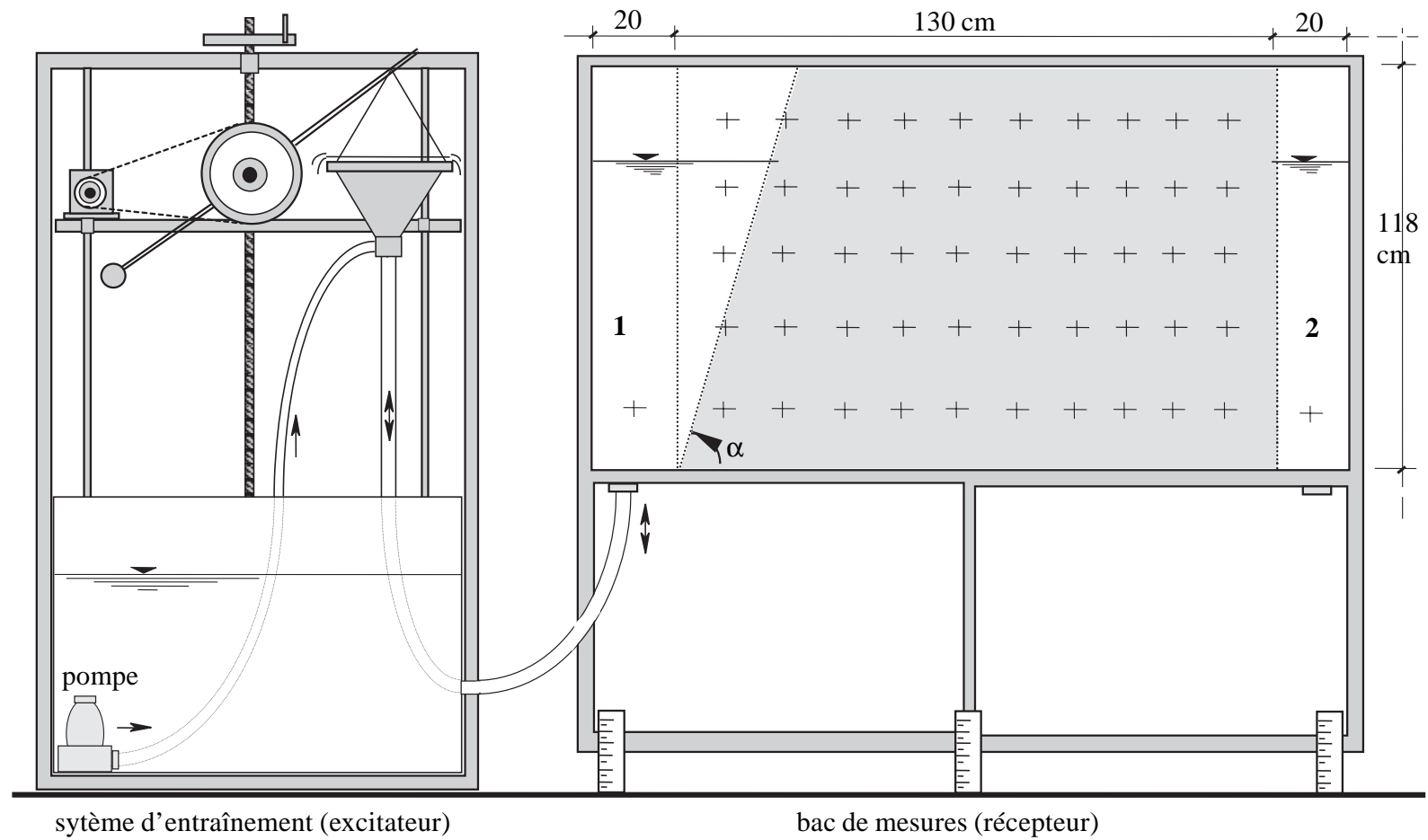

Fig. 8. Coupe schématique du remblai du site des Sablières (stratigraphie indicative).

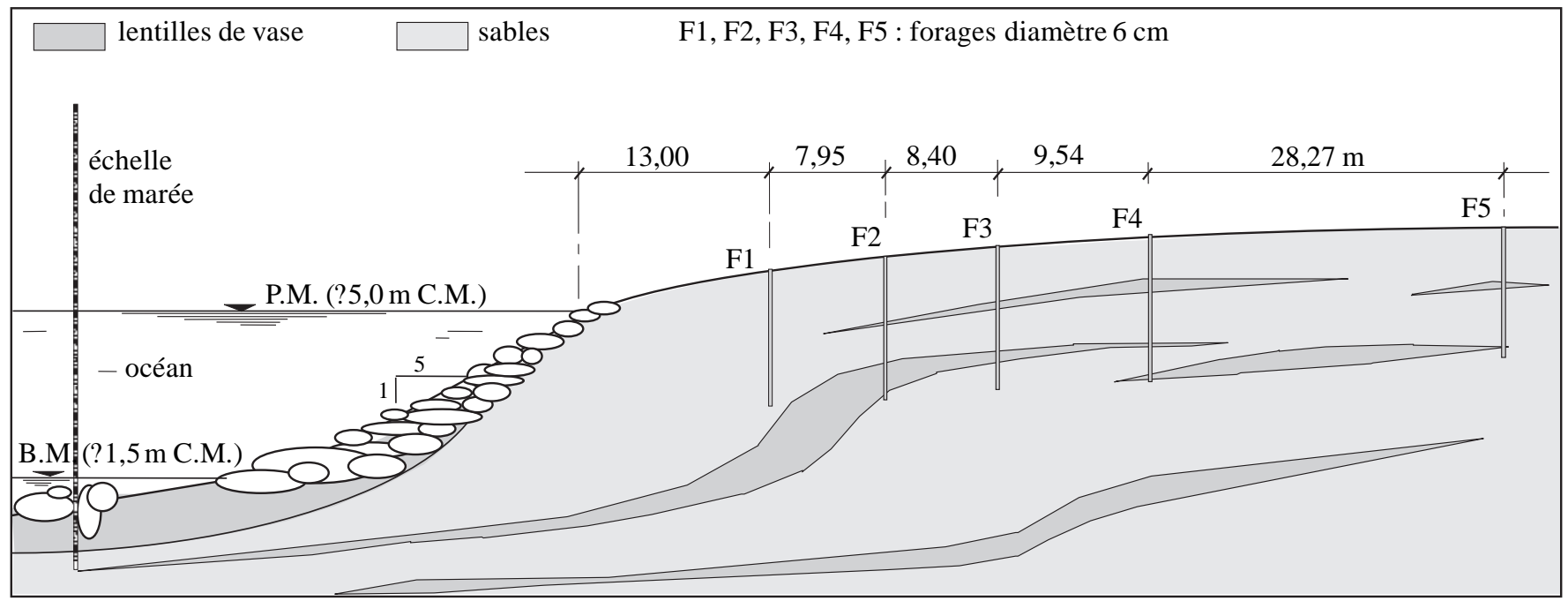

cycliques ont alors conduit à vérifier si les résultats originaux des modélisations précedentes étaient confirmés par des expérimentations sur un ouvrage réel.

\section{Choix et instrumentation d'un site réel}

Un site estuarien (les Sablières, rive Nord de la Loire, France), ayant une géométrie simple et des conditions aux limites aisément identifiables, a été choisi. Il s'agit d'un talus de remblai montrant des dépôts successifs de couches de sable de compacité moyenne et de vase (fig. 8). La pente (environ 1/5) est protégée par un enrochement en pierres naturelles. Le talus est alternativement complètement immergé à pleine mer, et complètement émergé à basse mer. La basse mer découvre un fond vaseux traversé par des résurgences et des trous d'affouillement d'environ $50 \mathrm{~cm}$ de diamètre, à travers lesquels le talus se vide pendant le jusant.

Pour effectuer le suivi de la surface libre pendant le cycle de marée, cinq forages ont été réalisés (F1 à F5), d'environ 2,5 $\mathrm{m}$ de profondeur sur le site, sur une ligne perpendiculaire à la rive.

\section{Confrontation théorie-expérimentation}

Confrontation entre expérimentation sur modèle physique et simulation numérique

Les résultats d'un essai en laboratoire ont été confrontés avec ceux de la simulation correspondante. 
Fig. 9. Comparaison simulation - modèle physique.

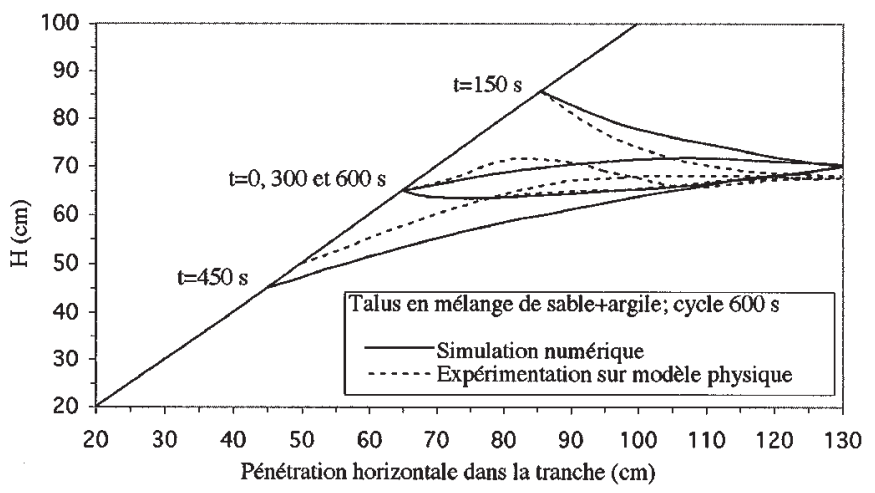

Les conditions d'essai sont $T=600 \mathrm{~s} ; H_{\text {moy }}=64,9 \mathrm{~cm}$; $A=20,1 \mathrm{~cm}$; pente $1 / 1$; sol constitué de sable $(0 / 1 \mathrm{~mm})$ additionné de $10 \%$ d'argile avec une perméabilité $1,8 \times 10^{-5}$ $\mathrm{m} / \mathrm{s}$. Dans ce cas (fig. 9) le niveau d'équilibre atteint $67,6 \mathrm{~cm}$, alors que la prévision par [4] est de 69,9 cm. Dans le cas limite du massif vertical $\left(T=600 \mathrm{~s} ; H_{\text {moy }}=70,4 \mathrm{~cm}\right.$; $A=21,1 \mathrm{~cm}$ ) le niveau d'équilibre atteint $72,7 \mathrm{~cm}$, alors que la prévision est de $73,4 \mathrm{~cm}$. Un accord global entre les niveaux piézométriques calculés et mesurés étant constaté (fig. 9), la modélisation paraît donc satisfaisante.

\section{Confrontation entre expérimentation sur site et simulation numérique}

La confrontation entre l'expérimentation sur modèle physique et la simulation numérique doit être complétée par une comparaison entre une expérimentation sur site et la simulation numérique, en raison de la complexité des phénomènes naturels qui ne peuvent être qu'incomplètement pris en compte par la modélisation numérique.

Les mesures ont été relevées en période sèche et de faibles coefficients de marée (fin juin 1993), le long d'un cycle de marée (coefficient de marée 44; basse mer : 1,75 m C.M. à $8 \mathrm{~h} 17$; pleine mer : 4,20 m C.M. à 14h36) où les hauteurs sont exprimées en cotes marines (C.M.) par rapport au zéro hydrographique.

Du fait de l'hétérogénéité du massif, la perméabilité et la porosité locales ne peuvent être représentatives. Nos mesures de perméabilité in situ au moyen des essais Lefranc par injection à niveau variable ont permis d'estimer les caractéristiques globales à : $k=2,6 \times 10^{-4} \mathrm{~m} / \mathrm{s}$ et $n=0,2($ Rezzoug 1994).

La figure 10 indique les mesures de niveau d'eau effectuées pendant le cycle de marée en joignant simplement tous les points de mesures. Le système de coordonnées est composé de la hauteur verticale mesurée en mètres C.M. et de la distance mesurant la pénétration horizontale dans le remblai. Les résultats sont représentés à chaque quart du cycle : miflot $(t=0)$, pleine mer $(t=3 \mathrm{~h})$, mi-jusant $(t=6 \mathrm{~h})$ et basse $\operatorname{mer}(t=9 \mathrm{~h})$.

Les profils de surface libre (fig.10) montrent : $(i)$ des évolutions similaires entre les profils mesurés et simulés (zone battue, niveau d'équilibre, forts rabattements à marée basse); (ii) une longueur de zone battue expérimentale supérieure à la longueur simulée; (iii) un niveau d'équilibre mesuré supé-
Fig. 10. Comparaison simulation - expérimentation in situ.

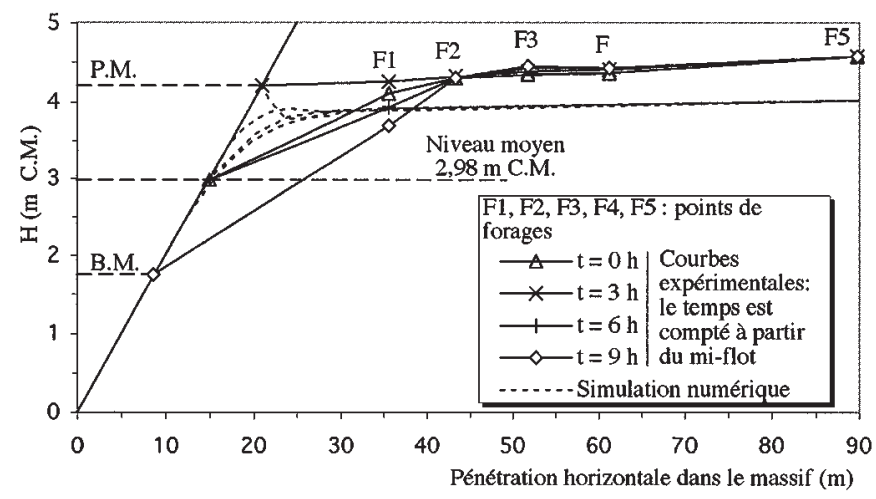

rieur au niveau simulé, ces deux niveaux restant toutefois voisins du niveau de pleine mer.

La surélévation du niveau d'équilibre par rapport au niveau moyen apparaît dans les deux approches théorique et expérimentale in situ.

Le manque de concordance entre ces deux niveaux ne peut être attribué à la capillarité dans la zone non saturée, car elle n'apporte pas de modification significative aux résultats (Rezzoug 1994).

Les écarts entre les courbes théoriques et expérimentales peuvent s'expliquer par :

- L'hétérogénéité des sols à l'origine d'imprécisions dans l'estimation de la perméabilité représentative : un calcul avec une perméabilité de 2,5 fois la valeur estimée (soit 6,5 $\times 10^{-4} \mathrm{~m} / \mathrm{s}$ ) a permis une bonne simulation de la longueur de la zone battue mesurée.

- La représentation simplifiée de la marée en une marée semi-diurne : un calcul avec un coefficient de marée de 75 (coefficient moyen) a permis une bonne simulation du niveau d'équilibre mesuré. Ceci tendrait à montrer une inertie importante du massif vis-à-vis de la marée, et que le niveau d'équilibre serait plutôt conditionné par la période lunaire de la marée (28 jours).

- Les apports latéraux possibles : on peut toutefois remarquer que dans cette région et en période sèche les apports d'eau salée semblent nettement supérieurs aux apports latéraux d'eau douce (aquifère de faible puissance). Le phénomène hydraulique prédominant conditionne le niveau d'équilibre et réduit ainsi l'influence de ces apports latéraux possibles.

\section{Conclusion}

L'étude de l'écoulement à surface libre dans un massif soumis à la marée a été réalisée en associant théorie, calcul, et expérimentations en laboratoire et in situ.

La démarche adoptée repose sur les points suivants : $(i)$ proposition d'une modélisation basée sur l'équation de Dupuit en régime transitoire non linéaire, (ii) réalisation d'expérimentations en laboratoire au moyen d'un modèle physique original, (iii) réalisation d'expérimentations sur site, (iv) confrontation entre simulations et expérimentations.

Cette démarche a permis : $(i)$ des simulations numériques par deux méthodes différentes (différences finies et développement de Fourrier) cohérentes avec un résultat analytique 
important; (ii) une bonne validation de la simulation des essais de laboratoire sur modèle physique, on a pu ainsi cerner les causes des imprécisions de la simulation des essais en nature.

Les principaux résultats obtenus sont : $(i)$ le bon accord global entre les trois approches : simulation, expérimentation en laboratoire et in situ, (ii) la présence d'une zone battue, lieu de fortes vitesses d'écoulement, (iii) l'existence d'un niveau d'équilibre dans le massif, supérieur au niveau moyen de la marée et proche du niveau de pleine mer pour les talus naturels.

La prise en compte de la marée de période lunaire (bien qu'elle multiplie le temps de calcul par environ 60) ainsi que l'étude de l'influence de l'écoulement engendré par la marée sur la stabilité des talus intertidaux semblent constituer des perspectives intéressantes à partir de cette étude.

\section{Bibliographie}

Alexis, A. 1987. Étude géotechnique et sédimentologique de souilles et chenaux de la rade de Lorient. Contribution à la stabilité des talus immergés. Thèse de docteur ingénieur. École nationale supérieure de mécanique, Nantes, p. 260.

Boussinesq, J. 1904. Recherches théoriques sur l'écoulement des nappes d'eau infiltrées dans le sol et sur le débit des sources. Revue de mathématiques appliquées, 1 : 7-79.

Castany, G. 1967. Traité pratique des eaux souterraines. Édition Dunod, Paris.

Chapon, J. 1966. Travaux maritimes. Tome 2. Édition Eyrolles, Paris.

Dagan, G. 1967. Linearized solutions of free surface ground-water flow with uniform recharge. Journal of Geophysical Research, 72: 1183-1193.

De Cazenove, E. 1971. Ondes phréatiques sinusoïdales. La houille blanche, 7 : 601-615.

Desai, C.S. 1970. Analysis transient seepage using viscous flow model and numerical methods. Seepage in Mississipi River banks. Rapport 1. Février 1970. National Technical Information Service, U.S. Department of Commerce.
Desai, C.S. 1971. Unconfined transient seepage in sloping banks. Proceedings of the American Society of Civil Engineers, Journal of Soil Mechanics and Foundations Division, 97(SM2) : 357373.

Hall, K., Smith, G.M., and Turcke, D.J. 1994. Development of a non-linear porous media flow relationship for oscillatory unsteady flow. Journal of Coastal Research, 10(1) : 158-169.

Humbert, P. 1984. Application de la méthode des éléments finis aux écoulements en milieu poreux. Digues et barrages en terre de faible hauteur. Bulletin de liaison des laboratoires des ponts et chaussées, 132 : 21-37.

Marino, M.A. 1973. Water table fluctuation in semipervious steamunconfined aquifer systems. Journal of Hydrology, 19 : 43-52.

Ould Amy, M. 1990. Modélisation numérique des écoulements et des déformations dans les barrages en terre construits sur des sols mous. Thèse de doctorat de l'École nationale des ponts et chaussées, Paris, France.

Polubarinova, P.Y. 1962. Theory of ground water movement. Princeton Ed., Princeton, N.J.

Rat, M., et Vautrin, J. 1973. Détermination des caractéristiques hydrauliques des sols à partir des variations piézométriques. Revue de liaison du laboratoire des ponts et chaussées, 1280 : 103-119.

Razack, M., Drogue, C., Romariz, C., et Almeida, C. 1980. Étude de l'effet de la marée océanique sur un aquifère carbonaté côtier. Journal of Hydrology, 45 : 57-69.

Rezzoug, A. 1994. Influence de la marée sur un sol semi-immergé. Thèse de doctorat de l'École centrale et de l'Université de Nantes, Nantes, France.

Rezzoug, A., Alexis, A., et Thomas, P. 1994a. Simulation expérimentale de l'écoulement dans un talus de sol soumis à la marée. Revue française de géotechnique, 69 : 31-39.

Rezzoug, A., Alexis, A., et Thomas, P. 1994b. Écoulement dans les talus intertidaux. Journal de recherche océanographique. 19(3/4) : 125-136.

Vauclin, M. 1975. Étude expérimentale et numérique du drainage de nappes à surface libre. Influence de la zone non saturée. Thèse de doctorat ès-sciences physiques, Université de Grenoble, Grenoble, France.

Zaradny, H. 1993. Groundwater flow in saturated and unsaturated soil. Edited by R.B. Zeidler. A.A. Balkema, Rotterdam. 